\title{
Assessing the Adequacy of Behavioral Health Training for Collegiate EMTs
}

\author{
Ali M. Baird \& Carol R.T. Day, RN, MSN, CNS
}

POSTER PRESENTATION ABSTRACT | ORIGINAL RESEARCH CATEGORY

Introduction: Increased prevalence and underreporting of mental health conditions on college campuses implies collegiate Emergency Medical Technicians (EMTs) may treat more patients with acute behavioral concerns. Georgetown Emergency Response Medical Service (GERMS), a collegiate based EMS (CBEMS) group, experienced a 450\% increase in behavioral calls from 2014 to 2016. This study aimed to assess EMT self-reported preparedness for behavioral emergencies obtained from the EMT course and continuing education provided by GERMS. A secondary aim was to compare behavioral call experience to preparedness.

Methods: In March 2018, 60 members of GERMS completed an online 14-question survey to assess preparedness and helpfulness of educational material about behavioral health training using 0 (meaning least) to 5 (meaning most) Likert scales and multiplechoice questions. All metrics from the survey are self-reported. Coverage of behavioral health topics in the EMT course, helpfulness of educational experiences about behavioral health provided by GERMS, and behavioral call experience were each compared independently to preparedness.

Results: All participants reported lower preparedness when they could not recall behavioral health topics covered in their initial EMT course as seen by an independent sample t test of differences between recall and preparedness for panic attacks (3.96 for recalled, 3.12 for unrecalled; $\mathrm{p}=0.002$ ), thoughts of self-harm without suicidal ideation ( 3.54 for recalled, 2.69 for unrecalled; $\mathrm{p}=0.005$ ), suicidal ideations ( 3.12 for recalled, 1.80 for unrecalled; $\mathrm{p}=0.004$ ), and suicide attempts $(3.13$ for recalled, 1.81 for unrecalled; $\mathrm{p}=<0.001$ ). Debriefs following behavioral emergencies was the only GERMS educational experience positively correlated with higher preparedness using Spearman's rho correlation. Using an independent sample t-test, behavioral call experience produced higher preparedness for all types of behavioral emergencies.

Discussion/Conclusions: Coverage of mental health topics in CBEMS groups is associated with perceived preparedness. CBEMS groups have an opportunity to improve behavioral health training to respond to the growing mental health crisis.

Author Affiliations: From Department of Human Science, School of Nursing and Health Studies, Georgetown University; Georgetown Emergency Response Medical Service - both in Washington, DC, USA (A.M.B.). From Department of Human Science, School of Nursing and Health Studies, Georgetown University; Georgetown Emergency Response Medical Service; Health Education Services, Georgetown University - both in Washington, DC, USA (C.R.T.D.).

Address for Correspondence: Ali Baird | Email: alimbaird@me.com

Conflicts of Interest/Funding Sources: By the JCEMS Submission Declaration Form, all authors are required to disclose all potential conflicts of interest and funding sources. The authors declared that they have no conflicts of interest. The authors declared that they did not receive funding to conduct the program or research associated with this work.

Ethical Compliance: The authors attest that the research associated with this abstract was conducted in accordance with the JCEMS Ethics Guidelines.

Submission History: Received December 8, 2019; accepted for presentation and publication January 8, 2020.

Poster Presentation: This abstract was presented as a poster at the Academic Poster
Session of the 27th Annual Conference of the National Collegiate Emergency Medical Services Foundation; February 29, 2020; Boston, MA, USA.

Published Online: December 31, 2020

Published in Print: December 31, 2020 (Volume 3: Issue 2)

Reviewer Information: In accordance with JCEMS editorial policy, poster presentation abstracts undergo double-blind peer-review by at least two reviewers (JCEMS Editorial Board members and/or independent reviewers) prior to acceptance for presentation and publication. JCEMS thanks the anonymous reviewers who contributed to the review of this work.

Copyright: $\odot 2020$ Baird \& Day. This is an open access article distributed under the terms of the Creative Commons Attribution 4.0 International (CC BY 4.0) License, which permits unrestricted use, distribution, and reproduction in any medium, provided the original author and source are credited. The full license is available at: https://creativecommons.org/licenses/by/4.0/

Electronic Link: https://doi.org/10.30542/JCEMS.2020.03.S1.04 